J. Clin. Chem. Clin. Biochem.

Vol. 14, 1976, pp. 181-183

\title{
Atomabsorptionsspektrometrische Bestimmung von Blei in Barthaaren
}

\author{
Von V. Graef \\ Zentrum für Biochemie der Universität Gießen
}

(Eingegangen am 27. Oktober/4. Dezember 1975)

Zusammenfassung: Der Bleigehalt der Haare aus dem elektrischen Rasierer kann mit der Mikrotiegel-Technik nach Delves atomabsorptionsspektrometrisch bestimmt werden. 1-5 $\mathrm{mg}$ der gewaschenen und getrockneten Haare werden mit Wasserstoffperoxid teilweise oxidiert und dann analysiert. Die Methode ist einfach und schnell durchzuführen und deshalb für Reihenuntersuchungen bleiexponierter Personen geeignet.

\section{Determination of lead in beard hair by atomic absorption spectrometry}

Summary: The lead content of hair from the electric razor can be determined by atomic absorption spectrometry, using the micro-sampling technique of Delves. $1-5 \mathrm{mg}$ of the washed and dried hair are partially oxidized with hydrogen peroxide prior to analysis. The method is simple and quick and therefore suited for the serial investigation of persons exposed to lead.

\section{Einführung}

Das durch Inhalation oder durch Nahrungsmittel aufgenommene Blei wird zu 90-95\% in den Knochen deponiert. Zwischen der Bleikonzentration des Blutes und dem Bleigehalt der Knochen besteht, jedenfalls bei einer chronischen Bleibelastung, keine eindeutige Korrelation $(1,2)$. Von Sterner (1) wurde aber eine gute Korrelation zwischen dem Bleigehalt der Knochen und dem Bleigehalt der Haare festgestellt. Von Schübel et al. (3) wurde eine Feldstudie über die Bleibelastung von Kindern durchgefuhit, wobei der Bleigehalt der Kopfhaare nach feuchter Veraschung durch Atomabsorptionsspektrophotometrie bestimmt wurde. Hierfür waren $0,5-1,0 \mathrm{~g}$ Haare erforderlich. Wir haben die Mikrotiegel-Technik von Delves (4) zur Bestimmung des Bleigehaltes von Haaren benutzt. Hierfüir sind nur 1-5 mg Haare pro Bestimmung erforderlich, eine feuchte Veraschung vor der Messung durch Atomabsorptionsspektrophotometrie ist nicht nötig. Zur Bestimmung verwendeten wir Barthaare aus dem Trockenrasierer.

\section{Material und Methoden}

\section{Reagenzien}

Blei(II)-nitrat p.a., Perhydrol p.a. 30\% : Fa. E. Merck, Darmstadt; Dodecylsulfat-Natriumsalz : Fa. Serva, Heideliberg.

\section{Geräte}

Atomabsorptions-Spektralphotometer EEl 240 (Fa. Evans Electroselenium Ltd. Halstead, Essex, England). Die Zusatzeinrichtung für die Mikrotiegel-Technik und die Mikro-Nickeltiegel stammen von der gleichen Firma. Servogor-Schreiher RE $511: \mathrm{Fa}$ Metrawatt.

\section{Vorbereitung der Haare}

Die Haare, die sich nach mehreren Rasuren im Trockenrasierer angesammelt haben, werden $15 \mathrm{~min}$ mit etwa $10 \mathrm{ml}$ NatriumDodecylsulfat-Lösung $(20 \mathrm{~g} / \mathrm{l})$ in einem Zentrifugenröhrchen behandelt. Nach kurzer Zentrifugation wird die weiße Schicht (Hautpartikel) zwischen Haaren und überstehender Lösung mit einem Glasstab aufgewirbelt, die wäßrige Lösung wird abgegossen und verworfen. Die Haare werden anschließend noch zweimal je $5 \mathrm{~min}$ mit Natrium-Dodecylsulfat-Lösung behandelt, die weiße Zwischenschicht wird ebenfalls aufgewirbelt, worauf die überstehende Lösung abgegossen wird. Dann werden die Haare abfiltriert, reichlich mit bidest. Wasser und Äthanol gewaschen und auf dem Filter über Nacht bei $110^{\circ} \mathrm{C}$ im Trockenschrank getrocknet. 1-5 mg Haare werden in einem Mikro-Nickeltiegel mit $20 \mu$ Perhydrol etwa $1 \mathrm{~min}$ auf der Heizplatte bei etwa $150^{\circ} \mathrm{C}$ erwärmt und dadurch vorverascht. In einem weiteren Tiegel wird die gleiche Menge Haare mit $20 \mu$ B Bleinitrat-Lösung $(1 \mu \mathrm{mol} / \mathrm{l} \mathrm{Pb})$ auf der Heizplatte bei $150^{\circ} \mathrm{C}$ eingedampft und in gleicher Weise mit $20 \mu \mathrm{l}$ Perhydrol vorverascht. Neue Nickeltiegel müssen vor ihrer ersten Verwendung vorbehandelt werden. In ihnen werden $20 \mu \mathrm{l}$ Blut mit $20 \mu \mathrm{l}$ Perhydrol bei $150^{\circ} \mathrm{C}$ auf der Heizplatte erwärmt. Sie werden dann in der Flamme des Atomabsorptionsspektrophotometers ausgeglüht.

Atomabsorptionsspektrometrische Bestimmung des Bleis Wir benutzten die Mikrotiegel-Technik nach Delves $(4,5)$, bei der auf dem schlitzförmigen Brenner (Acetylen, Luft) des Atomabsorptionsspektrophotometers ein Nickel-Absorptionsrohr (Länge $11 \mathrm{~cm}$, Durchmesser 1,3 cm) befestigt wird, das an der 


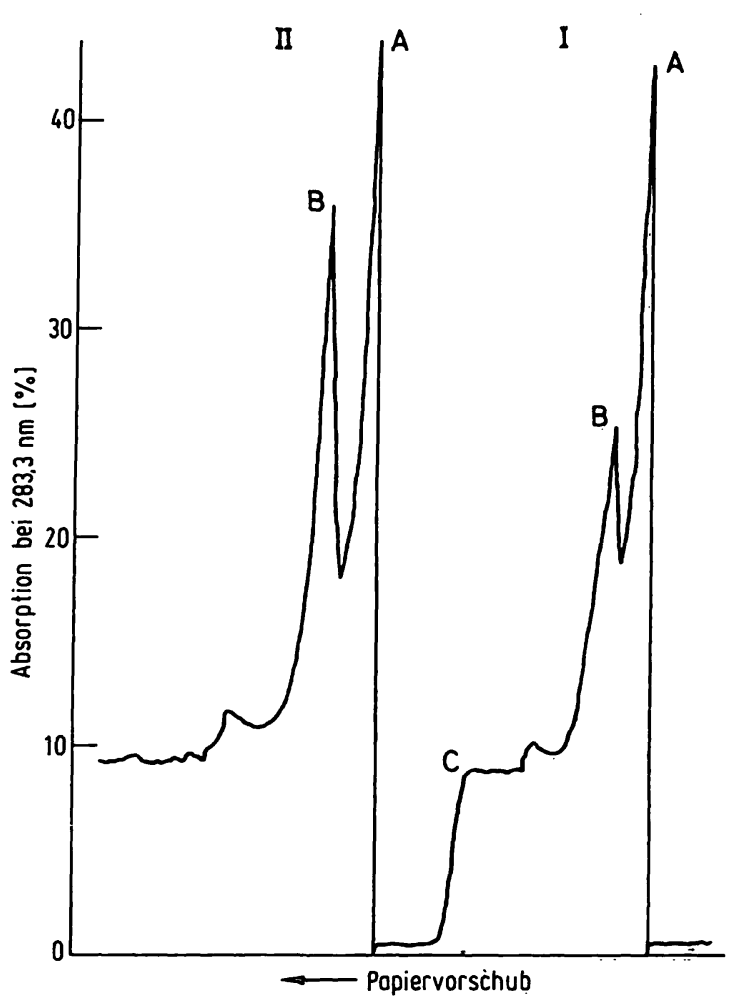

Abb. 1. Bleibestimmung in Haaren ohne (I) und mit (II) Bleizusatz; $A=$ Rauchpeak; $B=$ Bleipeak; $C=$ Entfernung des Tiegels aus der Flamme.

unteren, dem Brenner zugewandten Seite eine Öffnung besitzt. Mit einer besonderen Vorrichtung wird ein Mikrotiegel mit der vorbereiteten Probe in diese Öffnung eingeführt. Dabei verdampft das im Tiegel enthaltene Blei und die Bleidämpfe fuillen kurzfristig das Absorptionsrohr. Die Messung erfolgt mit der Absorptionslinie des Bleis bei $283,3 \mathrm{~nm}$. Nach Einbringen des Tiegels mit der Probe in die Flamme registriert der Schreiber einen Doppel-Peak: zuerst wird der Rauchpeak registriert, diesem folgt der Peak des Bleis (Abb. 1). Man wartet ab, bis der Schreiber die Grundlinie erreicht hat und entfernt dann den Tiegel aus der Flamme. Die Peakhöhe von dieser Grundlinie bis zum Peakgipfel wird abgemessen und dient zur Berechnung des Bleigehaltes der Probe. Wenn der Schreiber Absorptionswerte registriert, werden diese nach der Formel $E=2-\log (100-A \%)$ in Extinktionen umgerechnet. Zur Berechnung des Bleigehaltes der Haarprobe wird die Extinktionsdifferenz zwischen Haarprobe mit und ohne Bleizusatz benutzt. Der Bleigehalt der Haare beträgt

$$
\frac{20 \cdot \mathrm{E}_{\mathrm{H}}}{\left(\mathrm{E}_{\mathrm{HZ}}-\mathrm{E}_{\mathrm{H}}\right) \cdot \mathrm{G}} \mu \mathrm{mol} / \mathrm{kg}
$$

$\mathrm{E}_{\mathrm{H}}=$ Extinktion der Haare ohne Bleizusatz, $\mathrm{E}_{\mathrm{HZ}}=$ Extinktion der Haare mit Bleizusatz $(20 \mu \mathrm{l}$ einer Bleilösung von $1 \mu \mathrm{mol} / 1$ $\mathrm{Pb}), \mathrm{G}=$ Gewicht der Haare (mg).

\section{Ergebnisse und Diskussion}

\section{Linearität}

Die Linearität wurde geprüft, indem Proben von je $2 \mathrm{mg}$ Haaren im Tiegel mit je $20 \mu$ l Bleinitrat-Lösung steigender Konzentration versetzt und in der beschriebenen Weise gemessen wurden. Die Standardkurve war linear bis zu einer absoluten Bleimenge von $120 \mathrm{pmol}$ in der Probe (Abb. 2). Auch mit steigenden Mengen ( $1-4 \mathrm{mg}$ ) der

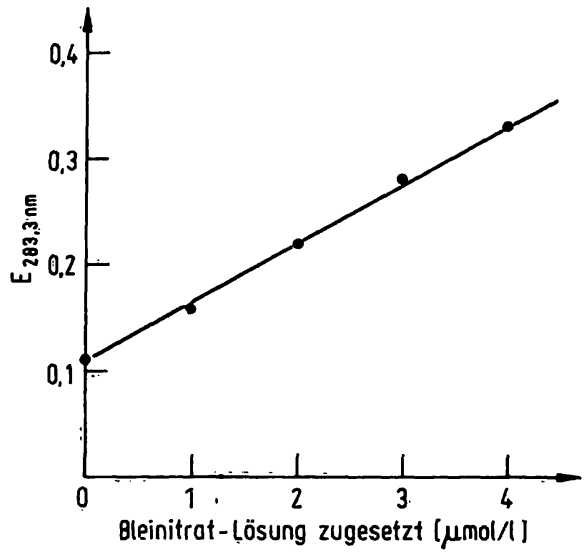

Abb. 2. Bleibestimmung in einer Haarprobe $(2 \mathrm{mg})$ ohne und mit Zusatz von $20 \mu$ l verschieden konzentrierter Bleilösungen.

gleichen Haarprobe, die $8, \overline{7} \mu \mathrm{mol} / \mathrm{kg} \mathrm{Pb}$ enthielt, ergab sich eine Linearität.

\section{Präzision}

Zur Bestimmung der Präzision wurde eine Haarprobe $20 \mathrm{mal}$ analysiert. Der Mittelwert betrug $8,45 \mu \mathrm{mol} / \mathrm{kg}$, die Standardabweichung 0,69 $\mu \mathrm{mol} / \mathrm{kg}$ und der Variationskoeffizient $8,1 \%$.

\section{Wiederfindung}

Zur Prüfung der Richtigkeit der Methode wurden 10 Haarproben (je $2 \mathrm{mg}$ ) mit einem Bleigehalt von 10,7 $\mu \mathrm{mol} / \mathrm{kg}$ mit $20 \mu \mathrm{l}$ einer Bleinitrat-Lösung $(2 \mu \mathrm{mol} / \mathrm{l})$ versetzt, in der angegebenen Weise verascht und anschließend gemessen. Zum Vergleich wurden je $2 \mathrm{mg}$ Haare von jungen weißen Mäusen, die kein Blei enthielten, mit der gleichen Menge Bleinitrat-Lösung versetzt. Die Wiederfindung betrug 100,4 $\pm 12,8 \%$.

\section{Bleigehalt von Barthaaren}

In Barthaaren von 20 gesunden Männem, die keiner Bleibelastung ausgesetzt waren, fanden wir 10,1 \pm $4,77 \mu \mathrm{mol} / \mathrm{kg} \mathrm{Pb}(\overline{\mathrm{x}} \pm \mathrm{s})$. Hammer et al. (6) fanden in den USA in Kopfhaaren nach feuchter Veraschung höhere Werte. Personen in Städten ohne Industrie hatten einen Bleigehalt in Haaren von durchschnittlich $31 \mu \mathrm{mol} /$ $\mathrm{kg}$. In Tabelle 1 ist der Bleigehalt der Barthaare einiger Männer angegeben, die beruflich mit Blei in Berührung

Tab. 1. Bleigehalt von Barthaaren bleibelasteter Personen.

\begin{tabular}{lc}
\hline Beruf $\cdots$ & $\begin{array}{l}\mathrm{Pb} \text { in } \\
\text { Barthaaren } \\
(\mu \mathrm{mol} / \mathrm{kg})\end{array}$ \\
\hline Buchdrucker (Handsetzer) & 134 \\
Gießer (kommt mit Pb-Dämpfen in Berührung) & 70,5 \\
Tankwart & 26,6 \\
Tankwart & 41,3 \\
Berufskraftfahrer & 26,6 \\
Berufskraftfahirer & 24,6 \\
\hline
\end{tabular}


kommen. Ihr Bleigehalt liegt über den Werten, die bei Normalpersonen gefunden wurden. Die Bleikonzentration des Blutes des Buchdruckers lag mit 1,36 $\mu \mathrm{mol} / 1$ an der oberen Grenze des Normalbereichs.

Bei früheren Methoden zur Bestimmung des Bleigehaltes in Haaren wurde die feuchte Veraschung mit Salpetersäure angewandt (1). Hierfür sind größere Mengen Haare erforderlich. Einfacher und schneller läßt sich die Bleibestimmung in Haaren durchführen, wenn man die Haare nach kurzer Behandlung mit Perhydrol in Mikrotiegeln direkt in die Flamme eines Atomabsorptionsphotometers einführt. Hierfür sind nur wenige mg Haare erforderlich. Bei Verwendung vọn Kopfhaaren müssen diese fein zerschnitten werden. Ein weiterer Nachteil der Bleibestimmung in Kopfhaaren besteht darin, daß kopfferne Partien der Haare zu einer Zeit gewachsen sein können, in der die Person nicht mit Blei in Berührung kam; in diesen Abschnitten der Haare würde man weniger Blei finden. Durch Verwendung von Barthaaren aus dem Trockenrasierer hat man die Gewähr dafür, daß diese Haare die Bleiexposition der letzten Tage wiederspiegeln. Barthaare fallen außerdem in feiner Form an, sie brauchen nicht zerkleinert zu werden.

Es kann ausgeschlossen werden, daß äußerliche Bleispuren an den Haaren mitgemessen werden, da die Haare mit einem Detergenz und mit Alkohol gründlich gewaschen werden. Es wurde festgestellt (7), daß äußerliche Schwermetallspuren durch Waschen entfernt werden.

Wir fanden, daß zur schnellen Verbrennung der Haare in der Flamme kleine Mengen Eisen nötig sind. Deshalb haben wir in neuen Nickeltiegeln vor dem Gebrauch Blut verbrannt. In neuen, nicht vorbehandelten Tiegeln, erhielten wir breitere Peaks mit einer geringeren Peakhöhe.

Durch eine Bleibestimmung in Haaren kann eine Bleispeicherung aufgedeckt werden. Die beschriebene Methode könnte für epidemiologische Fragestellungen auf dem Gebiet der allgemeinen Ökotoxikologie bedeutungsvoll sein. Sie ist so einfach durchführbar, daß es möglich ist, die Bleibelastung einer größeren Anzahl von Personen in kurzer Zeit festzustellen.

\section{Literatur}

1. Sterner, W. (1972). Arch. Lebensmittelhyg. 23, 209-213.

2. Reif, E., Post, D., Graef, V., Steioff, W., Tiedtke, K. H.,

Rutenfranz, J. \& Sbresny, W. (1974). Öff. Gesundh.-Wesen 36, 787-798.

3. Schübel, E., Schübel, R. \& Sterner, W. (1973). Deut. Ärztebl. 2194-2199.
4. Delves, H. T. (1970). Analyst 95, 431-438.

5. Heinemann, G. (1973), diese Z. 11, 197-201.

6. Hammer, D. I., Finklea, J. F., Hendricks, R. H., Shy, C. M. \& Horton, R. J. (1971). J. Amer. Med. Ass. 215, 384-385.

7. Yamaguchi, S. \& Matsumoto, H. (1966). Proc. $15^{\text {th }}$ Internat. Congr. Occup. Health, Wien, S. 255.

\section{Dr. V. Graef}

Zentrum f. Biochemie am Klinikum d. Justus Liebig-Univ. 63 Gießen

Friedrichstr. 24 
